\title{
Un « volume » incertain : géologie politique de l'exploration du gaz de charbon en Lorraine (France) ${ }^{\star}$
}

\author{
Olivier Labussière * \\ Géographie, CNRS, UMR PACTE, Université Grenoble-Alpes, SciencePo Grenoble, Grenoble, France
}

\begin{abstract}
Résumé - Cet article suit les prospections de gaz de charbon entreprises par une compagnie junior australienne, Kimberley Oil, dans le Bassin lorrain entre 2000 et 2015. Cette compagnie procède par forages horizontaux dans des couches de charbon naturellement fracturées. Ces développements visent à produire du gaz en France et à réduire les émissions de $\mathrm{CO}_{2}$ liées aux importations gazières. L'article s'inscrit dans le champ de la géologie politique. Il mobilise la notion de « strate» développée par Nigel Clark pour porter un propos de sciences sociales sur les processus de recomposition des sous-sols en contexte de transition. Il étudie l'émergence d'une nouvelle strate (gazière) en tenant compte de l'ensemble des relations qui la constituent et qui sont opérées dans l'incertaine matérialité d'un volume. À travers l'étude de ces relations, il contribue à éclairer la formation de nouvelles segmentations autant géologiques que sociales, et interroge le contenu politique de cette promesse de transition.
\end{abstract}

Mots-clés : gaz de charbon / exploration / strate / volume / géologie politique / France

\begin{abstract}
An uncertain "volume" : political geology of the coalbed methane exploration in Lorraine (France). This article follows the coalbed methane exploration undertaken by an Australian junior company, Kimberley Oil, in the Lorrain Basin between 2000 and 2015. This company is proceeding by horizontal drilling in naturally fractured coal seams. These developments aim to produce gas in France and to reduce $\mathrm{CO}_{2}$ emissions by substituting local gas for foreign imports. The article falls within the field of political geology. It mobilizes the notion of 'stratum' developed by Nigel Clark to bring a social science perspective to the processes of subsoil recomposition in a context of transition. It studies the emergence of a new 'stratum' (coalbed methane), taking into account all the relations that constitute it and that are operated in the uncertain materiality of a volume. Through the study of these relations, it contributes to shedding light on the formation of new segmentations, both geological and social, and questions the political content of this promise of transition.
\end{abstract}

Keywords: coalbed methane / exploration / stratum / volume / political geology / France

Le fort développement des gaz non conventionnels, aux États-Unis puis en Europe, au début des années 2000 a bousculé les scénarios de transition, ouvrant ceux-ci à la possibilité d'une étape intermédiaire de décarbonisation, en substituant le charbon par le gaz (IEA, 2012). Le gaz se trouve au cœur des scénarios de transition en ce

\footnotetext{
‡ Cet article est une contribution au numéro coordonné par X. Arnauld de Sartre et S. Chailleux, intitulé «Pour une géologie politique ».

*Auteur correspondant :

olivier.labussiere@umrpacte.fr
}

qu'il est à la fois moins "carboné » que le charbon et qu'il participe d'une stratégie de stockage des surplus électriques issus des énergies renouvelables ${ }^{1}$. Cette étape intermédiaire permet à tout un secteur industriel de se prévaloir d'une contribution à la lutte contre le changement climatique au travers de la construction

\footnotetext{
${ }^{1}$ Les surplus électriques issus des énergies dites renouvelables peuvent être utilisés pour produire de l'hydrogène $\left(\mathrm{H}_{2}\right)$ à partir de l'électrolyse de l'eau, ensuite stocké et injecté dans un réseau de distribution de gaz naturel. L'hydrogène produit peut aussi être associé à du dioxyde de carbone $\left(\mathrm{CO}_{2}\right)$ par méthanation afin de produire un méthane de synthèse $\left(\mathrm{CH}_{4}\right)$.
} 
de fuel bridges ${ }^{2}$ controversés (Delborne et al., 2020), vers une économie bas carbone.

Cet article suit les prospections de gaz de charbon entreprises par une compagnie junior australienne, Kimberley Oil, dans le Bassin lorrain entre 2000 et 2015. La distinction entre les gaz de schiste, dont le développement a connu un coup d'arrêt en raison de l'interdiction de la fracturation hydraulique en 2011, et le gaz de charbon est ici importante. Kimberley Oil procède par forages horizontaux dans des couches de charbon «naturellement» fracturées. L'article étudie le développement de cette clean-tech qui vise à réduire les émissions de dioxyde de carbone $\left(\mathrm{CO}_{2}\right)$ par substitution de gaz local à des importations.

Cet article mobilise la notion de «strate» (Clark, 2016) pour porter un propos de sciences sociales sur les processus de recomposition des sous-sols en contexte de transition. Il étudie l'émergence d'une nouvelle strate (gazière) en tenant compte de l'ensemble des relations qui la constituent et qui sont opérées dans l'incertaine matérialité d'un «volume» (Serres, 1977). À travers l'étude de ces relations, il contribue à éclairer la formation de nouvelles segmentations autant géologiques que sociales et interroge le contenu politique de cette promesse de transition.

\section{Une proposition de géologie politique}

\section{Aborder le sous-sol comme une strate}

En sciences sociales, le sous-sol et ses ressources (gaz de schiste, gaz de charbon) sont régulièrement abordés au travers des mobilisations sociales que leur exploitation suscite. La sociologie argumentative étudie la façon dont des acteurs sociaux font évoluer leurs argumentaires afin d'étoffer leurs alliances et d'orienter le cours d'un projet (Chateauraynaud et Debaze, 2017). L'analyse des emplois sémantiques (le gaz de charbon, le gaz de houille, le méthane) permet d'identifier les éventuelles collusions entre les différentes parties impliquées (industriels, experts, scientifiques) (Stein, 2019). L'analyse des politiques publiques montre l'importance de l'usage politique des connaissances dans la bataille entre experts et contre-experts, par exemple au sujet de l'interdiction de la fracturation hydraulique en France (Baudrin et al., 2014; Chailleux, 2019).

\footnotetext{
2 La notion de fuel bridge appartient à un argument d'ensemble qui vise à ménager les filières industrielles carbonées en différant les coûts liés au déploiement des énergies renouvelables. Cela consiste à introduire dans les scénarios de transition un palier intermédiaires de décarbonisation par le recours transitoire mais important à des hydrocarbures moins émetteurs de $\mathrm{CO}_{2}$.
}

Cet article emprunte une voie différente, en proposant de prendre pied dans le champ de la géologie politique. Celle-ci vise à dépasser un partage épistémologique implicite qui cantonnerait les sciences sociales à l'analyse des passions et des représentations sociales, en «surface», tandis que les sciences de l'univers s'attacheraient à l'étude de la nature des «profondeurs». Cet article défend la possibilité de porter un propos de sciences sociales sur les sous-sols, c'est-à-dire sur les processus d'exploration et de requalification dont ils sont l'objet en contexte de transition vers un monde bas carbone. Cet abord ne réduit pas le sous-sol à un construit sociopolitique: la terre est belle et bien porteuse d'une part «inconstructible» (Neyrat, 2017).

Le géographe Nigel Clark (2013) proposa la notion de «strate» pour interroger ce partage épistémologique. Parler de strate consiste à aborder la terre comme une entité sociopolitique sans exclure ses dynamiques intrinsèques. Cette proposition émergea dans un contexte précis, celui d'un intérêt grandissant de la géographie politique pour les nouvelles formes de contrôle étatique de l'espace terrestre par une gestion en «volume» (les tunnels sous les frontières: Elden, 2013; les enjeux environnementaux globaux: Dalby, 2013). Clark introduisit la notion de strate pour marquer une différence avec celle plus classique de territoire. Le territoire pense l'homme comme un facteur politique majeur de transformation de l'espace. Cette lecture anthropocentrée perd de sa pertinence à mesure que l'espace des préoccupations politiques s'étend au terrestre. Elle tend à survaloriser la capacité de contrôle des organisations sociopolitiques sur des entités (les plaques tectoniques dans le cas des séismes) qui, par leurs échelles, leurs temporalités, leurs matérialités les dépassent et leur résistent.

Clark édifie la notion de strate sur des bases deleuziennes (Clark, 2016). Une strate est un principe de double articulation entre des hétérogènes, humains et terrestres. Les relations de l'humain au terrestre ne sont pas données, elles sont prises dans des processus de stratification et de destratification qui adviennent dans des sites. Ces strates sont des sites de mise en politique de la terre. La géologie politique, au-delà d'un auteur, est aujourd'hui un champ qui prend de l'ampleur (Bobbette et Donovan, 2019) - tout comme la géographie politique approfondit l'idée d'une gouvernance étatique de l'espace en volume (Billé, 2020).

\section{À quoi tient une strate?}

Dans cet article, la notion de strate est mobilisée pour aborder l'activité minière dans ses rapports à des couches géologiques spécifiques. Les mines de houille en Lorraine constituent un cas exemplaire de formation 
d'une strate productive-non pas seulement au sens d'une épopée socio-économique, mais bien d'une entreprise collective d'articulation au terrestre. Pour extraire la houille, il fallait être en capacité de suivre une veine de charbon, d'assurer l'exhaure minier ou encore d'éventer le grisou.

La venue au début des années 2000, dans un contexte d'après-mine, d'une junior entreprise à la recherche de gaz de charbon constitue un cas intéressant de constitution d'une nouvelle strate productive, gazière cette fois-ci. Une lecture anthropocentrée de l'exploitation minière pourrait suggérer que la strate houillère ayant perdu sa vocation économique, elle retournerait à l'état de nature. Si elle a perdu le collectif sociotechnique qui la rendait productive, elle n'est pas inerte pour autant (mouvements de terrain, remontée de la nappe phréatique) et continue de susciter de nombreux concernements sociaux (dommages sur les maisons, transformations paysagères, administration des risques). Le cas lorrain suggère qu'une strate houillère en voie de déstratification interagit avec une strate gazière en voie de stratification. L'œuvre de Clark invite à étudier la façon dont des strates se refont les unes sur les autres, mais il ne précise pas la façon d'enquêter sur ces interrelations.

Pour surmonter cette difficulté, je propose de mobiliser la notion de «volume» que Clark avait préalablement écartée au motif qu'elle véhiculerait une lecture territoriale et anthropocentrée du politique. Pour cela, je la revisite à l'aune du travail de Michel Serres (1977) sur l'atomisme de Lucrèce : si la pensée classique nous a habitués à voir un volume comme une idéalité géométrique qui valorise la maîtrise de l'homme sur la nature, celle de Lucrèce insiste davantage sur les dynamiques d'émergence aussi bien matérielles que sociopolitiques dont un volume procède. Le volume est ici un moyen d'enquêter sur les liens constitutifs d'une strate, en prêtant attention à la façon dont ils sont opérés dans la densité de la matière.

Le travail de Michel Serres sur Lucrèce n'a pas qu'une utilité de circonstance. Il propose aussi une façon originale de réassembler deux courants de travaux en sciences sociales sur la notion de volume : l'une valorise l'attention pour des matérialités changeantes (volumineux), l'autre insiste sur les puissances qui organisent l'espace comme un volume contrôlable (volumétrique).

\section{Un ensemble de relations prises dans l'incertaine matérialité d'un volume}

Un premier ensemble de travaux porte sur le volumineux, c'est-à-dire les qualités changeantes d'un médium en transformation. Il est possible de rapprocher ces travaux, en géographie, de l'attention portée aux approches relationnelles de l'espace (Whatmore, 2002; Massey, 2005). L'espace n'est pas un contenant passif au sein duquel les relations sociales prennent place. Il présente des qualités liées aux médiums qui le constituent. Steinberg et Peters (2015) prennent l'océan comme cas paradigmatique: son volume n'est pas d'abord une volumétrie, mais un ensemble de molécules qui change constamment de relations, de vitesses, de formes à mesure que différentes forces (courants marins, vent, soleil) influent sur ses changements d'état (solides, liquides ou gazeux). D'autres travaux tout en conservant cette part élémentaire ouvrent la notion à l'expérience humaine. Le corps constitue un moyen de se rendre sensible à un volume, d'en découvrir les dimensions matérielles, sensibles et affectives (Adey, 2013; McCormack, 2015; Squire, 2017). Cette perspective me permettra dans la suite de l'analyse de souligner combien, en situation de forage, les géologues adaptent leurs compétences à la matérialité des strates qu'ils contactent.

Un deuxième ensemble de travaux lie la notion de volume aux enjeux de connaissance, de pouvoir et de propriété. La dimension verticale de l'espace a été abordée pour analyser les liens historiques entre la construction de la science géologique et celle de l'État (Braun, 2000). La notion de volume est mobilisée en géographie politique pour intégrer les enjeux contemporains d'extensions du contrôle territorial. Prenant le cas de la frontière entre Israël et le Liban, Elden (2013) analyse la façon dont différentes infrastructures et environnements rendent la frontière poreuse à des flux de biens, d'armes et de personnes. Ce travail en a inspiré d'autres sur la prolifération des métriques destinées à gérer l'environnement global (Dalby, 2013). Dans le champ de la géographie des ressources, la notion de volume a été introduite comme a spatial form of property through which the circulation of resources and commodities is controlled (Bridge, 2013). Gouverner par les volumes ne doit toutefois pas réduire la question de la mise en politique de la terre à la toute-puissance de l'humain sur la nature, à l'image de la géo-ingénierie (Lehman, 2013).

L'œuvre de Michel Serres, La naissance de la physique (1977), offre une troisième voie qui permet de faire dialoguer ces deux dimensions, le volumineux et le volumétrique. L'auteur propose une lecture de l'œuvre de Lucrèce, De la nature (Lucrèce, 2002). À la différence de la pensée classique qui conçoit la nature comme un ensemble régi par des lois, la physique de Lucrèce met l'accent sur une nature dont «la stabilité d'ensemble n'est que la résultante des instabilités particulières » (Gigandet, 2002: 31). Cette nature n'est pas linéaire et prédictible, mais tourbillonnaire et laisse place à 
l'émergence. La course générale des éléments, observe Serres, ne cesse de fluctuer entre deux formes tourbillonnaires: «La première désigne une multitude, une grande population, la confusion et le tumulte. C'est le désordre [...] Mais le second est une forme ronde en mouvement comme un sabot ou comme la toupie, cône qui tourne ou spirale tourbillonnaire [...] Le monde en sa globalité peut être modélisé par tourbillons. L'origine des choses et le commencement de l'ordre consistent simplement dans ce passage fin entre turba et turbo [...] Le premier demeure un désordre et le deuxième une certaine forme en mouvement» (Serres, 1977: 38-39).

Un volume n'est pas défini ex ante, il doit sa constitution à la course spiralée des éléments. Il résulte de la transformation progressive d'une «multitude» (de molécules, de connaissances, de passions politiques) en «une certaine forme en mouvement». Cette idée est importante, car elle n'assujettit pas l'élémentaire (le volumineux) à une forme abstraite (le volumétrique); au contraire, un volume est une forme émergente et précaire. Le volumineux et le volumétrique sont deux dimensions d'un même volume, en tant que forme toujours stable et instable à la fois, se faisant et se défaisant.

\section{Une enquête pragmatiste sur les relations constitutives d'une strate}

La notion de volume permet d'enquêter sur les relations constitutives d'une strate. Le volume est l'espace concret au sein duquel des médiations vont être testées, hiérarchisées, négociées afin qu'une strate productive soit constituée. En ce sens, un volume est une figure de potentiel permettant d'ouvrir les sous-sols à des intérêts productifs; c'est là une façon parmi d'autres de potentialiser le sous-sol ${ }^{3}$.

Cette enquête s'inscrit dans une approche pragmatiste des processus contemporains de transition énergétique (Labussière et Nadaï, 2018). La contribution de ces processus aux enjeux de transition n'est pas donnée. Leur potentiel est en voie de construction au fur et à mesure du déploiement de nouvelles technologies de l'énergie et de la structuration de nouveaux collectifs sociotechniques. Ces potentiels de transition suscitent des troubles ontologiques (Marres, 2012) appelant à réévaluer collectivement la valeur de nos relations à l'espace, aux paysages ou encore aux animaux. Ces troubles ne sont pas toujours correctement pris en charge ou adossés à des publics (Dewey, 1927), et peuvent susciter de

\footnotetext{
3 D'autres approches valorisent moins la capacité à créer des relations dans la densité de la matière que l'habitabilité du sous-sol (Adey, 2013) ou encore sa capacité à dissimuler des déchets (Hamblin, 2008).
}

nouvelles injustices sociales et environnementales au nom de la transition.

Cet article est issu du projet Gazhouille mené en collaboration avec des chercheurs en géologie et en sciences sociales (2013-2015). Les chercheurs en géologie étaient étroitement associés au projet d'exploration du gaz de charbon mené par European Gas Limited $^{4}$. Cette situation a facilité la rencontre des experts de la compagnie et la compréhension des enjeux auxquels ils se sont affrontés sur le terrain. L'enquête a aussi été élargie à d'autres parties prenantes telles que l'administration régionale, des associations et des habitants. Dans l'ensemble, j'ai réalisé vingt entretiens avec, par répartition d'enjeux: (i) explorer: les géologues experts et ceux chargés du forage, en bureau et sur site lors d'une visite de la plateforme de Tritteling; (ii) modéliser: l'ingénieur des mines en charge de la collecte des archives des Houillères des bassins de Lorraine (HBL), le géologue en charge de la reconstitution paléogéologique du Bassin lorrain, le responsable du département modélisation; (iii) partager : le responsable d'une agence régionale de protection de l'environnement, une association d'opposants au gaz de charbon, l'autorité régionale pour le développement économique, celle pour la protection de l'environnement, une membre du Schéma d'aménagement et de gestion des eaux (Sage) $\mathrm{du}$ bassin houiller, un élu local et des chercheurs en géologie. J'ai également analysé des rapports d'enquête publique. L'analyse étudie la fabrique d'un volume de gaz de charbon (explorer, modéliser, partager) et la façon dont ces relations émergentes configurent politiquement une nouvelle strate productive.

\section{La fabrique d'un volume: une enquête multiscalaire}

L'industrie du charbon a connu des développements considérables dans le Bassin lorrain durant plus d'un siècle et demi (Fig. 1). Après la Seconde Guerre Mondiale, l'État français a nationalisé cette industrie, avec la création de Charbonnage de France et de sa branche régionale, les Houillères des bassins de Lorraine (HBL). Cette strate minière gouvernée par une compagnie publique d'État déclina à partir des années 1960 , jusqu'à l'arrêt de la dernière mine en 2004. La fermeture des HBL suscita une nouvelle donne territoriale favorable à l'arrivée d'intérêts industriels étrangers.

\footnotetext{
4 Avant 2006, la compagnie s'appelle Kimberley Oil (KO). Elle est renommée European Gas Limited (EGL) en janvier 2006 et Française de l'énergie (FDE) en juin 2015. Ces changements témoignent de son ambition croissante pour exploiter un champ gazier, et non plus seulement le prospecter, et de ses efforts pour apparaître comme une entreprise qui contribue à la souveraineté énergétique de la France.
} 


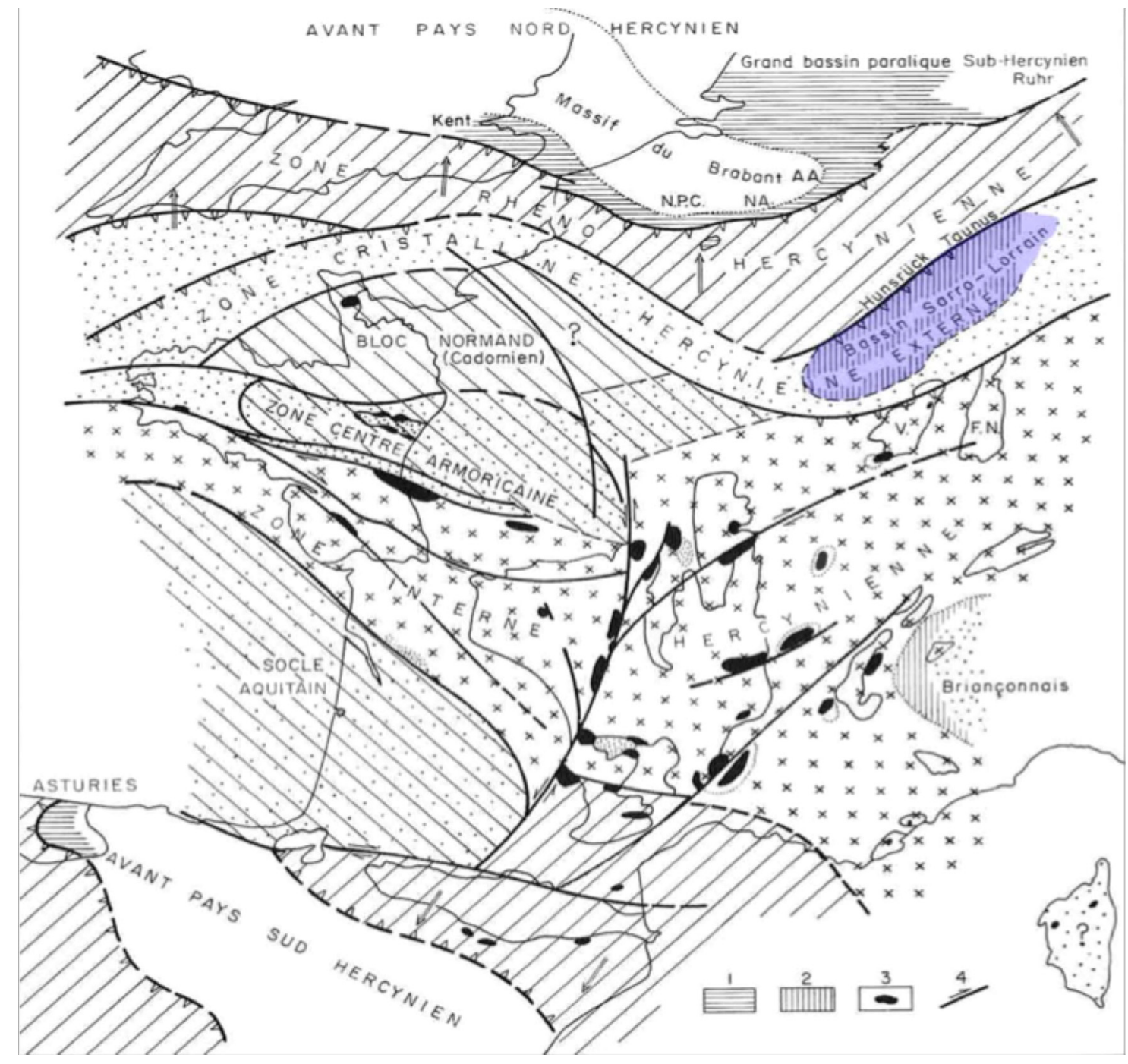

Fig. 1. Localisation du Bassin sarro-lorrain (fond de carte Donsimoni, 1981).

Cet article étudie la façon dont une strate gazière se constitue sur une ancienne strate minière de 2000 à 2015. La ressource ciblée n'est alors plus le minerai de charbon, mais des molécules de méthane diffuses piégées à la surface des charbons.

Le passage d'une ressource à une autre est un vrai défi pour l'administration du sous-sol. En France, le sol et le sous-sol appartiennent au propriétaire du terrain. Des exceptions encadrées par le Code des mines adopté en 1810 permettent à l'État de délivrer des permis miniers à des fins d'exploration ou d'exploitation des substances minérales du sous-sol. Alors que les autorisations sont délivrées suivant une liste de substances, ce procédé ignore la matérialité des gisements et les moyens techniques de leur exploitation. À la différence de la prospection des gaz de schiste, Kimberley Oil procède par drains horizontaux dans des couches de charbon naturellement fracturées; ce qui fait du cas lorrain un cas rare en France d'exploration toujours en cours d'un gaz non conventionnel.

\section{Former un concept géologique, explorer plus qu'un volume}

Cette première ligne d'enquête suit des géologues et des foreurs, au travers d'une succession d'expériences menées dans le temps (des années 1990 à 2015) et l'espace (France, Canada). L'analyse montre comment ces expériences contribuent à forger leur compétence d'exploration des charbons humides du Bassin lorrain. Chaque expérience est guidée par un «concept géologique », c'est-à-dire une hypothèse de connectivité entre des entités géologiques (mort-terrain, veines de charbon, failles, aquifère) partiellement connues dans leur extension, leur densité ou leur porosité. L'enjeu d'opérer ces connexions dans la densité de la matière est d'ouvrir 
Tab. 1. Évolution des expérimentations conduites par EGL sur trois sites pilotes (Folschwiller-1, 2 et Tritteling-1) (réalisation: O. Labussière, à partir d'entretiens auprès d'ingénieurs d'EGL).

\begin{tabular}{|c|c|c|c|}
\hline Sites pilotes & Folschwiller-1 & Folschwiller-2 & Tritteling-1 \\
\hline Compétences & $\begin{array}{l}\text { Ingénieurs retraités des HBL, } \\
\text { mobilisation de savoir-faire } \\
\text { hérités pour déterminer où et } \\
\text { comment forer dans le Bassin } \\
\text { lorrain }\end{array}$ & $\begin{array}{l}\text { Ingénieurs expérimentés venant } \\
\text { de l'Alberta, mobilisation de } \\
\text { savoir-faire issus du monde } \\
\text { pétrolier/gazier en matière de } \\
\text { forage horizontal }\end{array}$ & $\begin{array}{l}\text { Améliorer le pilotage de la tête } \\
\text { de forage (agréger les données, } \\
\text { visualiser les couches) }\end{array}$ \\
\hline $\begin{array}{l}\text { Matérialité, } \\
\text { circulation }\end{array}$ & $\begin{array}{l}\text { Reconstitution sans fin d'un } \\
\text { volume d'eau par la faille } \\
\text { interceptée }\end{array}$ & $\begin{array}{l}\text { Récupération limitée d'un volume } \\
\text { de gaz }\end{array}$ & $\begin{array}{l}\text { Production d'un volume } \\
\text { irrécupérable constitué d'une } \\
\text { mixture de gaz et d'eau }\end{array}$ \\
\hline
\end{tabular}

le sous-sol à passage, pour créer une circulation de molécules de méthane du fond vers la surface.

Un «volume», dit Serres, est une forme en mouvement qui ordonne la «multitude». À l'image d'une toupie oscillant sur son axe qui explore un espace plus large que son corps, les travaux de forage suscitent des connexions inattendues dans l'étendue du sous-sol. Plus que de simples variations dans les routes que suivent les molécules, ces changements affectent la façon dont celles-ci s'agrègent et changent d'état. En retour, ces connexions fortuites appellent à modifier l'assemblage sociotechnique du forage tout entier (les hommes, les gestes, les techniques, les instruments). En suivant les évolutions de «concept géologique» (Tab. 1), l'analyse montre l'étroite relation entre l'exploration de la matière (volumineux) et l'émergence de métriques empiriques (volumétriques).

À son arrivée en Lorraine, Kimberley Oil n'a que peu de connaissances de la géologie du bassin. Elle recrute en 2005 un ingénieur des HBL à la retraite pour pallier son déficit d'expertise. Celui-ci collecte les archives des HBL et parvient à s'orienter dans cette multitude de connaissances grâce à son expérience de la mine. Il identifie sur le plan de mine de Folschwiller une veine de charbon non exploitée avec un fort potentiel en gaz (Fig. 2). Grâce à sa compréhension intuitive de l'espace minier, il arrête la localisation du premier point de forage - ni trop près ni trop loin : «Il ne faut surtout pas s'approcher des vieux travaux, si on fore là-dedans, on n'a que de l'eau, on n'a pas de gaz ${ }^{5}$.»

En 2007, European Gas Limited (EGL) recrute l'ancien chef du service sondage des HBL pour conduire les premiers forages d'exploration, à Folschviller (FOL-1) et Diebling (DIE-1). Le «concept» est d'atteindre une faille afin de désorber plus facilement le gaz. À Folschwiller (puits FOL-2), des morts-terrains contraignent la trajectoire du forage qui n'aboutit au placement que d'un drain horizontal (A). Il intercepte malgré tout la couche Maurice Michel jusqu'à «taper» dans une faille: «On pensait qu'on aurait un "soufflard" [une sortie brutale de gaz], mais on n'a pas eu de gaz du tout, que de l'eau'.» L'équipe de forage réalise que cette faille est alimentée par une nappe phréatique. We were pumping the ocean ${ }^{7}$ ! Dans ces charbons humides, les failles jouent un rôle aléatoire de circulation des éléments du sous-sol (eau, méthane). Le forage est suspendu.

En 2008, EGL recrute deux foreurs canadiens provenant de l'Alberta. La priorité est de sauver le puits

\footnotetext{
${ }^{5}$ Entretien \#3, FDE, 15 octobre 2015 .

${ }^{6}$ Entretien \#2, EGL, 20 mai 2015.

${ }^{7}$ Entretien \#2, EGL, 20 mai 2015.
} 


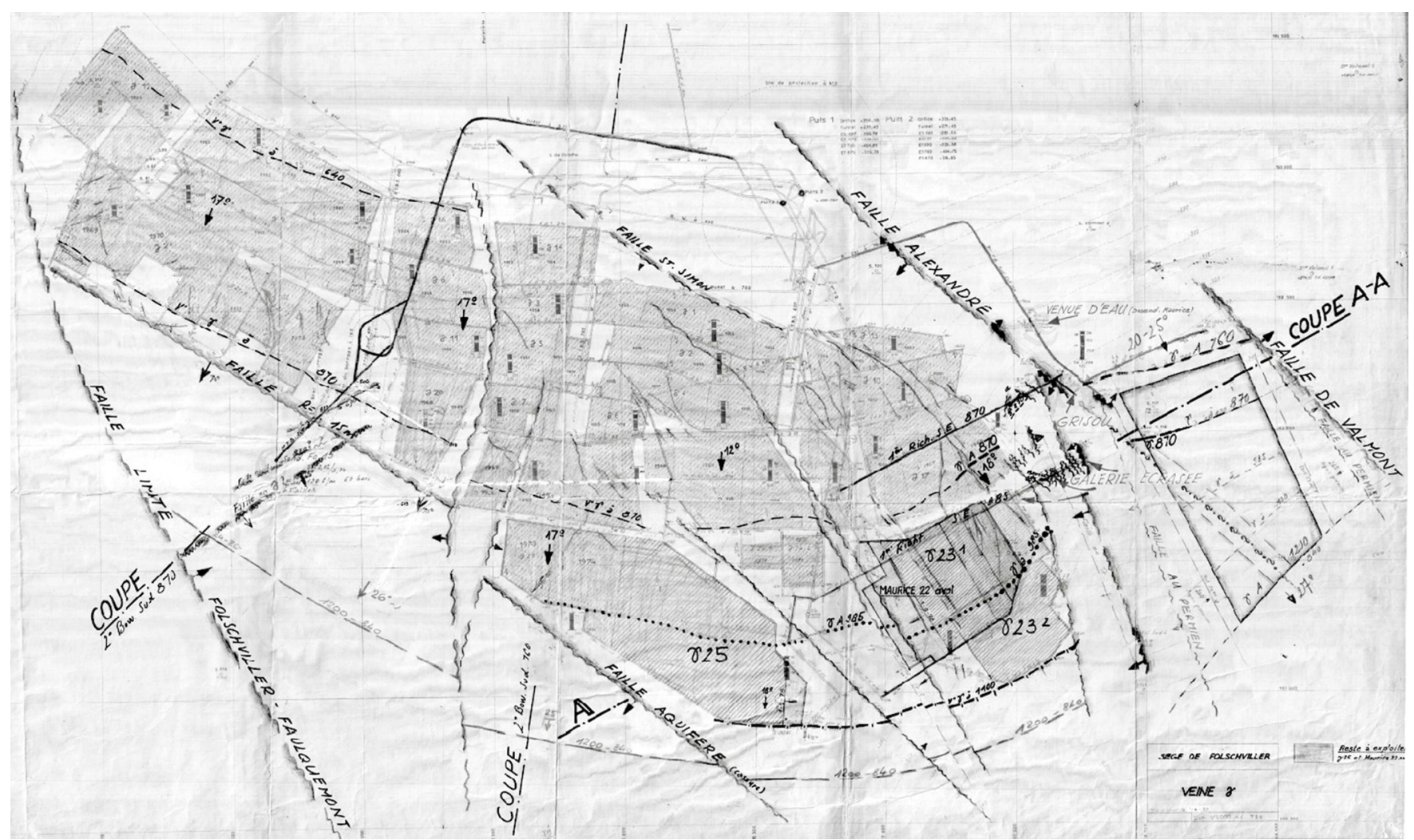

Fig. 2. Plan de la mine de Folschviller, veine de charbon $\gamma$ (source : Plan de la mine de Folschviller, veine de charbon $\gamma$, Houillères des Bassins de Lorraine, 1978). Le panneau de charbon inexploité est représenté par un quadrilatère blanc à l'extrême est de la carte.

FOL-2 et d'élaborer un concept géologique adapté aux charbons humides. L'équipe en place ne parvenait plus à situer précisément la tête de forage au fond du puits. Their ability to steer the drill was limited by the lack of experience. They have not appreciated the importance of bringing together all the available information ${ }^{8}$. Les foreurs canadiens améliorent le guidage du forage grâce à un logiciel, qui leur permet d'agréger en temps réel des données auparavant séparées (rayons gamma, vitesses de forage), et une habitude de travail en binôme sur plateforme qui minimise les erreurs de trajectoire. Malgré cela, ils ne parviennent pas à isoler le puits FOL-2 du drain ennoyé (A) et doivent abandonner la partie basse du puits, afin de créer de nouveaux drains horizontaux (B, C). The initial concept was three drains in three different seams (Maurice Michel, Alpha Beta Y, $\mathrm{Z}-\mathrm{Zi}$ ). This concept was complicated because the company wanted to do many experiments at the same time ${ }^{9}$. En 2010, l'opération réussit, mais les drains créés sont trop courts $(200 \mathrm{~m})$ et la quantité de gaz récupérée trop faible.

\footnotetext{
${ }^{8}$ Entretien \#2, EGL, 20 mai 2015.

9 Ibid.
}

En 2014, un nouveau puits d'exploration est ouvert à Tritteling. Le concept vise à placer des drains horizontaux plus longs dans un même panneau de charbon sans atteindre les failles qui l'interrompent. Six drains sont placés sur une longueur totale de 1500 mètres. Lors du test de production, une pompe est placée au point sécant des six drains pour retirer l'eau des charbons, puis entraîner le gaz à la surface. Un nouveau problème survient. L'action de la pompe remplit le forage d'une mixture de gaz et d'eau. Le gaz s'échappe par à-coups, fait tomber brutalement la colonne d'eau sur la vis de pompage et la brise. Au bureau, sur une étagère, trônent six trains de tige en acier cassés net. En octobre 2015, au terme de mes entretiens, ces tiges représentaient autant d'essais infructueux pour retirer le gaz de ces charbons humides. Le concept géologique allait à nouveau être modifié.

Cette première ligne d'enquête est riche d'enseignements. Tout d'abord, la capacité des géologues à lire une strate et à s'y orienter mobilise des connaissances incorporées (Rolston, 2013). L'ex-ingénieur des HBL sait s'orienter dans les plans de mine grâce à une vie consacrée au sous-sol lorrain. Les corps non reliés à cette strate minière se retrouvent perdus parmi une multitude 
de galeries et de vieux travaux. Il en est de même pour le pilotage de l'outil de forage. Les anciens mineurs ont une compétence limitée pour placer un forage horizontal dans des couches de charbon. Les foreurs canadiens sont plus expérimentés, bien qu'en Alberta leur expérience porte sur des charbons secs, horizontaux, à 400 mètres de profondeur, et non sur des charbons humides avec un pendage à $25^{\circ}$, à 1500 mètres. Leurs compétences doivent être réaccordées à la matérialité du sous-sol lorrain. Pour s'articuler à cette strate gazière en émergence, les foreurs ajustent entre eux des gestes et des instruments auparavant séparés (logs consolidés, visualisation en temps réel, synchronisation de l'interprétation et du pilotage). Par ailleurs, d'une expérimentation à l'autre, la géométrie du forage est précisée, le nombre des drains horizontaux accru et la distance entre les drains optimisée. Progressivement, les foreurs forment des métriques et une géométrisation du soussol étroitement liées aux premiers sites de forage. La production incessante d'eau ou la formation d'une mixture improductive d'eau et de gaz illustrent l'idée qu'un volume a toujours un surplus, des connexions avec quelque chose d'autre que la matérialité visée. Cette étape permet néanmoins à la compagnie d'estimer un premier volume de gaz récupérable et commercialisable.

\section{Calculer la ressource, modéliser moins qu'un volume}

Cette deuxième ligne d'enquête aborde le volume sous un angle différent. Elle suit les ingénieurs, les archivistes, les modélisateurs et les agents de certification au travail. Leurs efforts visent à calculer le volume de gaz récupérable sur l'ensemble du Bassin lorrain à partir des premiers forages d'exploration et d'archives minières. Ce passage du volume à l'échelle du bassin doit permettre d'attester de la réalité d'une strate productive profitable. Cette section montre que l'incertitude qui était le produit d'interactions hasardeuses avec le sous-sol est progressivement internalisée grâce au calcul d'une volumétrie de gaz, ce qui redispose la relation au sous-sol.

L'espace minier lorrain fut historiquement partagé entre plusieurs concessions (Petite-Rosselle, Sarre-etMoselle, Faulquemont-Folschviller). Chaque siège minier développa une connaissance géologique singulière. Ainsi, d'une mine à l'autre, une même veine de charbon pouvait ne pas porter le même nom ni être située à la même profondeur. Le bassin minier comprenait donc une collection de connaissances géologiques juxtaposées dans l'espace, mais non reliées entre elles. En 2005, Kimberley Oil passe « un accord avec les Houillères pour avoir accès aux archives. C'était une mine de renseignements $!{ }^{10}$. En quelques mois, une somme de données

$\overline{{ }^{10} \text { Entretien \#3, EGL, } 15}$ octobre 2015. publiques devient un actif stratégique pour cette compagnie privée, sans compensation financière, selon la volonté des HBL. La strate minière informe ici directement la strate gazière émergente.

Si l'ex-ingénieur des HBL parvient intuitivement à localiser un premier site de forage (voir section précédente), Kimberley Oil doit créer un modèle géologique global du Bassin lorrain pour évaluer la possibilité d'un champ gazier. À partir d'une multitude de données hétérogènes, la modélisation doit produire une représentation cohérente et unifiée du sous-sol. Le travail débute à l'automne 2011 par la reconstitution des veines de charbon afin de préciser leurs profondeurs, leurs pentes, leurs directions et leurs (dis)continuités. «Là [explique un modélisateur], on est dans un domaine lacustre avec des sortes de marécages. Et ces marécages vont former des plages à charbon qui vont évoluer en fonction du temps, du climat et de la tectonique en place. Les veines ne sont pas linéaires sur 200 mètres. Là, on en voit une qui se divise en trois plus petites ${ }^{11}$.» Le sous-sol lorrain devient un gigantesque puzzle dont les segments de charbon sont les pièces. Ces différentes pièces sont associées à des données d'âges différents : les archives minières des années 1950 (plans de mine, coupes stratigraphiques, données de concentration en gaz), les campagnes sismiques des années 1980 et les résultats des forages récents.

À partir de ce modèle, l'enjeu est de calculer la fraction de gaz récupérable, c'est-à-dire de préciser sa volumétrie en tenant compte de contraintes techniques, économiques et géographiques. En 2012, EGL demande la certification d'un expert indépendant (l'Institut français du pétrole) pour simuler son concept géologique sur une partie $(2,8 \%$ de la surface $)$ de ses permis d'exploration. EGL reçoit une certification de commercialité de la réserve gazière en avril 2012 et une mise à jour en septembre 2015 (IFP, 2015).

Le certificateur optimise l'architecture de puits utilisée à Tritteling (Trit-1, voir la section précédente) composée de quatre drains horizontaux espacés de 570 mètres. Il privilégie une géométrie standard de quatre drains espacés de 340 mètres, entourés d'un espace tampon (no flow boundary) de 170 mètres; la nappe phréatique est supposée n'avoir aucune incidence sur la production à cette distance. Bien qu'informée par le terrain, cette géométrie est à présent déconnectée d'une partie des données matérielles du sous-sol. Il cartographie aussi les terrains libres de contraintes urbanistiques et environnementales: cela aboutit à 39 forages potentiels intersectant 10 veines de charbon identifiées par le modèle géologique. Chaque intersection d'un puits avec une veine de charbon forme une aire de

$\overline{{ }^{11} \text { Entretien \#4, EGL, } 15}$ octobre 2015. 
production appelée «bloc». L'aire d'étude est divisée en 390 blocs considérés comme des unités indépendantes. L'exploitation d'un bloc est censée ne pas affecter les blocs voisins, verticalement et horizontalement. Cela signifie que les principes de connectivité et de circulation, qui étaient si difficiles à stabiliser sur le terrain du fait des interrelations aléatoires entre le méthane et l'eau, sont maintenant neutralisés. Chacun des 390 blocs est évalué individuellement (épaisseur, porosité, pression, contenu en gaz et en eau). L'estimation totale du gaz récupérable résulte de l'addition des valeurs de productivité de chaque bloc. Ainsi, la réserve gazière de $2,8 \%$ d'un permis détenu par EGL pour les 20 prochaines années est estimée à $5,3 \mathrm{Bcf}^{12}$ (prouvé), 27,9 Bcf (probable) et 73,5 Bcf (possible).

Les ressources en hydrocarbures non conventionnelles répondent à une norme internationale qui hiérarchise leurs potentiels selon le degré de certitude de leur production (Petroleum Resources Management System, 2011). Selon cette norme, le potentiel croît à mesure que l'on lève des barrières successives ${ }^{13}$. Contrairement à ce que suggère cette représentation de l'incertitude comme un continuum, l'analyse insiste sur le rôle de la matérialité et des échelles (celle du forage d'exploration, celle du champ gazier) dans le calcul d'un volume de gaz. À l'échelle d'un forage, ce présupposé d'homogénéité tombe et les pratiques de calcul doivent s'ouvrir à la matérialité du site. Une analyse fine des pratiques de calcul montre l'enjeu de la gestion de l'incertitude dans le passage d'un volume de gaz à l'échelle d'un bassin. L'incertitude est standardisée et distribuée au travers d'une nouvelle représentation du sous-sol organisée par blocs alignés verticalement et horizontalement. Elle n'est pas résorbée, mais placée dans les interstices. Ces interstices témoignent de la part inconstructible du soussol (failles, voies d'eau, morts-terrain). Le sous-sol est à présent formalisé comme une strate productive, c'est-àdire configuré comme une matrice de probabilités qui combine deux régimes d'incertitude liés à la petite échelle (matérialité changeante) et à la grande échelle (probabilité de production). Partager ce volume de gaz pour attirer des investisseurs et ordonner ses publics est au cœur de la prochaine section.

\footnotetext{
$\overline{12} 1$ Bcf correspond à 1 milliard de pieds cubes; 1 pied cube équivaut à 28 mètres cubes.

${ }^{13}$ Depuis l'évaluation du gaz physiquement présent dans une couche, le gaz productible sous certaines conditions technologiques, sous certaines conditions économiques, jusqu'au gaz qui a des probabilités d'être récupéré (réserves) et d'être effectivement produit (production).
}

\section{Ordonner les publics, partager un volume}

Cette dernière ligne d'enquête suit la façon dont un volume de gaz est publicisé et articulé à des publics hétérogènes. Elle observe les publicitaires, les investisseurs privés, les scientifiques, les administrations, les associations locales et l'assemblée de gestion de l'eau au travail. Elle interroge le contenu politique de ces articulations et la façon dont elle entend positionner cette strate au service d'une transition vers un monde bas carbone.

EGL doit lever des fonds afin de développer son activité d'exploration. En dépit d'un intérêt soutenu pour le projet lorrain, les investisseurs sont hésitants à investir dans un pays dont le gouvernement a bloqué net l'exploration des gaz de schiste en 2011. Our response was to say that the region and the state want the project to advance, and they will help financially, déclare le directeur d'EGL en février $2013^{14}$. La région Lorraine lutte depuis des décennies pour maintenir des emplois après des crises industrielles successives. Le passage du charbon au gaz est perçu par la collectivité régionale comme une voie prometteuse pour la croissance verte. Elle ne peut toutefois pas investir dans une aventure privée à risque. Elle propose donc à EGL et des chercheurs de l'université de Lorraine de porter un projet conjoint qui vise l'optimisation de technologies de récupération du méthane et l'appui du renouveau industriel régional. Il est finalement intégré à un plan régional d'ensemble (le Pacte Lorraine ${ }^{15}$ ) porté par l'État et la Région. Le projet renommé Regalor $^{16}$ (Ressources gazières de Lorraine) acquiert une dimension européenne (Luxembourg, Wallonie, Saar, Rhénanie-Palatine) courant 2014-2015. En juillet 2017, la Région Grand-Est attribue à Regalor une aide publique de 1,75 million d'euros, partagée entre la Française de l'énergie (FDE, ex. EGL) pour $500 \mathrm{k} €$ et l'université de Lorraine pour 1,25 M€. Ces soutiens publics participent de la stratégie d'ancrage d'EGL en France et de la mise en confiance de son actionnariat. Ils permettent aux chercheurs d'accéder via un puits foré par EGL à un pilote technologique pour leurs travaux sur l'usage du sous-sol pour une transition bas carbone (hydrogène, CCS, géothermie) ${ }^{17}$. Ces travaux, parce qu'ils sont stratégiques, restent confinés à une arène experte.

\footnotetext{
${ }^{14}$ Politics hamper bid to unlock French coal mine gas riches (https://www.reuters.com/article/us-france-gas-mines/poli tics-hamper-bid-to-unlock-french-coal-mine-gas-riches-idUS BRE91H0M320130218).

$15 \mathrm{http} / / /$ www.prefectures-regions.gouv.fr/content/download/ 14347/99771/file/PACTE Lorraine Document.pdf.

${ }^{16}$ Entretien \#4 avec un chercheur membre du projet Regalor, 21 mai 2015.

17 https://regalor.univ-lorraine.fr/folschviller/.
} 
À la suite d'estimations de ressource encourageantes, FDE envisage le développement d'un champ gazier, avec la mise en opération prochaine de nombreux forages ${ }^{18}$. Si une fraction de cette strate gazière (un puits) porte sur des approches bas carbone, la majeure partie reste articulée à l'économie usuelle des hydrocarbures. FDE lance une campagne publicitaire nationale ${ }^{19}$ pour lever des fonds privés en mars 2016. Le visuel montre des pots de confiture étiquettés «Gaz de houille de Lorraine» ou encore «Gaz de mine du Nord». Le gaz de charbon est présenté comme une commodité stable, alors que sa récupération est encore incertaine. Il est aussi associé à une tradition régionale, ce qui est discutable au regard de la strate en construction que cette exploration suscite. Cette campagne prépare l'entrée en bourse (Euronext, Paris) de la FDE au 30 juin 2016. Les «contenants» représentent sous la forme de petits volumes clos, facilement manipulables et appropriables, des parts d'actionnariat. C'est là une autre illustration de la façon dont un volume peut être publicisé et partagé avec des actionnaires privés sans rendre perceptible les incertitudes intrinsèques à son développement.

L'exploration du gaz de charbon reste peu débattue en Lorraine. Le point n'est pas mis à l'agenda de la concertation organisée pour l'élaboration du schéma régional climat, air, énergie en 2011-2012. Il est peu discuté par le comité chargé de l'élaboration du Sage du bassin houiller de 2008 à $2017^{20}$ : une seule fois, en mai 2013, à la suite de la présentation par EGL de son activité. Toutefois, le comité ne prend pas position par rapport au projet et ne le recense pas comme un enjeu du territoire $^{21}$. La fédération régionale de protection de l'environnement (Mirabel), qui regroupe 85 associations, dépose un recours contre l'autorisation préfectorale de quatre nouveaux forages d'exploration (Tritteling, Freybouse, Pontpierre et Louperhouse) en octobre $2013^{22}$. Elle exprime son inquiétude quant au risque grandissant de pollution de l'aquifère par une fuite de méthane dans le cas d'une exploitation à grande échelle. «Les grès du trias inférieur, c'est une immense réserve d'eau qui rejoint la Belgique, le Luxembourg, c'est une

\footnotetext{
$18 \mathrm{http} / / /$ www.francaisedelenergie.fr, rapport intitulé « document de base » produit pour l'Autorité des marchés financiers. EGL mentionne qu'à partir de 2018, la compagnie prévoit de passer d'une de prédevelopment à une phase de réplication.

19 Publicité visible à http://www.place-des-victoires.com/ project/francaise-de-lenergie.

20 https://www.gesteau.fr/sage/bassin-houiller.

21 Entretien \#5 avec la représentante d'une association nationale de défense des consommateurs, 19 juin 2015.

${ }^{22}$ Le préfet outrepassa l'obligation en vigueur depuis le $1^{\text {er }}$ juin 2012 d'organiser une enquête publique sur ces projets de forage.
}

ressource stratégique [...] l'idée d'EGL, c'est d'avoir des failles qui n'affectent pas le permo-triasique, alors que la majorité l'affecte [...] on a des risques de migration du méthane vers la nappe ${ }^{23}$.»

En 2015, la FDE dépose de nouvelles demandes d'autorisation de travaux miniers (Longeville, Zimming et Lachambre). La fédération régionale pointe le manque d'information de la part d'EGL: pourquoi multipler les forages ? Sont-ils encore exploratoires ou préfigurent-ils le démarrage d'une exploitation à grande échelle ${ }^{24}$ ? Sa contribution aux enquêtes publiques témoigne de sa préoccupation croissante pour la dégradation des paysages et des terres agricoles, ainsi que le risque de pollution de la nappe phréatique. À Longeville, l'enquête publique recueille 73 avis de particuliers et d'associations (1 pour, 72 contre). Le Sage du bassin houiller exprime des réserves: les mesures pour contrôler les travaux de forage et celles envisagées pour restaurer les sites ne sont pas assez détaillées. En dépit de ces éléments, le projet de Longeville reçoit un avis favorable du commissaire enquêteur, puis du préfet en novembre 2015. À Zimming, le commissaire enquêteur donne un avis défavorable en novembre 2015, suivant la position du conseil municipal et celle de la majorité des participants. Le préfet accorde malgré tout l'autorisation de travaux à la FDE en mars 2016. En décembre 2015 , les associations qui n'avaient pas pour habitude de manifester ensemble se rassemblent à Metz, puis à Saint-Avold en avril 2016.

Les mobilisations sociales portent sur des préoccupations (pollutions des eaux et des sols, mutations paysagères) qui ne répondent pas à la partition entre sous-sol, sol et territoire. Elles interrogent l'écologie des relations qui sous-tendent un volume en tant qu'entité géosociotechnique. Si ces relations problématiques suscitent de nouveaux publics - au sens de Dewey, elles restent pour autant indiscutées. Trois processus forgent l'ordre social de cette strate émergente: un néolibéralisme hybride qui procède par investissements croisés, public-privé, pour soutenir la croissance verte ; un appel public à capital qui ouvre le sous-sol lorrain à des investisseurs privés sans publiciser les incertitudes intrinsèques à l'exploration en cours; une tradition de gestion administrative du sous-sol qui du fait de son caractère très centralisé minore les concernements locaux et environnementaux.

\footnotetext{
${ }^{23}$ Entretien \#6 avec le directeur d'une fédération régionale de protection de l'environnement, 24 octobre 2013.

${ }^{24}$ Note, fédération régionale de protection de l'environnement, 14 octobre $2015,2 \mathrm{p}$.
} 


\section{Conclusion : les sous-sols comme nouvelles frontières politiques des processus de transition bas carbone}

La Française de l'énergie argumente qu'une molécule de méthane extraite du Bassin lorrain n'émet que 3,4 $\mathrm{g}$ de $\mathrm{CO}_{2} / \mathrm{kWh}$ contre $32 \mathrm{~g}$ pour une molécule importée par des fournisseurs gaziers étrangers. Sa stratégie s'inscrit dans une perspective de transition, à l'instar du positionnement récent des hydrocarbures non conventionnels comme de possibles fuel bridges vers un monde bas carbone. Le présent article propose une enquête pragmatiste pour analyser cette promesse de transition. Son originalité est de prendre pied dans le champ de la géologie politique afin d'appréhender le contenu politique de processus géosociotechniques, au-delà de la seule analyse des mobilisations sociales.

Un premier enseignement porte sur la notion de «strate» (Clark, 2016). L'émergence d'une strate productive (de gaz de charbon) peut facilement être confondue avec une couche géologique. Cet article a étudié la formation de cette strate à partir des relations qui sous-tendent sa structuration et qui sont à produire dans la densité de la matière, en volume. Cette perspective déplace les partages usuels entre sous-sol, sol et territoire pour attirer l'attention sur des processus plus transversaux. Le cas est éloquent à propos de la difficile séparation du méthane et de l'eau dans des charbons humides, mais de la très risquée migration de méthane dans la nappe phréatique. En termes de géologie politique, cette part terrestre, incertaine, est importante : elle rend compréhensible la difficulté et l'enjeu pour l'ensemble des groupes sociaux (foreurs, actionnaires, associations, administrations, habitants...) de s'articuler à elle, de la rendre partageable et gouvernable. Elle éclaire aussi les stratégies pour gérer l'incertitude et créer des formes de concernement différenciées.

Un deuxième enseignement porte sur le statut de l'incertitude. Celle-ci ne se réduit pas à un déficit de connaissances géologiques qu'il s'agirait d'acquérir. Elle est à la fois constitutive de l'expérience et présente un caractère transitif (Dewey, 1929). L'incertitude n'existe pas en soi, mais en relation avec les conditions d'une expérience donnée (constitutive), et l'expérience apparaît toujours incertaine au regard du potentiel qu'elle offre pour atteindre un état souhaité (transitive). Arraisonner les molécules de méthane a appelé une chaîne de médiations au travers de laquelle l'incertitude a été expérimentée (sur site), géométrisée (par des architectures de puits successives), circonscrite (par leur standardisation), distribuée dans les interstices (d'une matrice de blocs productifs), traduite en probabilités (par l'évaluation des catégories de ressource) et neutralisée (campagne publicitaire, arène experte, procédures administratives centralisées). Cette chaîne de médiations invite à penser qu'il s'agit moins d'un travail de réduction de l'incertitude que de distribution de celleci dans une formalisation du sous-sol pris comme une infrastructure de production ou strate. Ce travail a une dimension politique en ce qu'il vise à rassurer des investisseurs, à gagner des soutiens publics et à atténuer des oppositions locales.

Un troisième enseignement porte sur les fronts de transition et les nouvelles frontières qu'ils produisent. J'entends par fronts de transition la façon dont des espaces sont investis au nom de la transition par de nouvelles technologies de l'énergie et les recompositions politiques qui les sous-tendent. Le cas lorrain constitue une promesse de transition ambiguë en ce qu'elle prolonge l'exploitation d'hydrocarbures autant qu'elle prétend y mettre un terme. Elle tire parti de mécanismes publics de soutien à l'innovation dans une stratégie d'ancrage territorial qui s'avère opportuniste en ce qu'elle ne rend pas les publics informés de ses développements ni capables d'en négocier les orientations. C'est en ce sens que l'on peut parler de la naissance d'une nouvelle frontière: une nouvelle segmentation de l'espace du sous-sol (en une strate non reliée à la totalité des relations qu'elle engage) s'accompagne d'une nouvelle segmentation sociale (par l'absence de discussion sur l'écologie de relations d'une strate et le devenir des ressources du sous-sol).

\section{Remerciements}

Ce travail a bénéficié du soutien financier du CNRS, programme Gazhouille (2013-2015) coordonné par Y. Gunzburger. L'article a bénéficié d'échanges dans différents séminaires pour lesquels l'auteur remercie les personnes suivantes : université du Québec à Rimouski (M.-J. Fortin, Y. Fournis), « Journée sous-sols» (X.-A. de Sartre, P.-O. Garcia), AFSP 2017 session «Politiques énergétiques: modèles alternatifs et dynamiques locales » (S. Topçu, S. Chailleux).

\section{Références}

Adey P., 2013. Securing the volume/volumen: Comments on Stuart Elden's plenary paper 'secure the volume', Political Geography, 34, 52-54, https://doi.org/10.1016/j.pol geo.2013.01.003.

Baudrain M., Dauguet B., Deias D., Raimbault B., 2014. «On n'est pas des cow-boys ». Controverse sur l'exploitation des gaz de schiste et stratégie de l'industrie pétrolière, Revue d'anthropologie des connaissances, 8, 2, 451-478, https:// doi.org/10.3917/rac.023.0451.

Billé F., 2020. Voluminous States. Sovereignity, Materiality and the Territorial Imagination, London, Duke University Press. 
Bobbette A., Donovan A., 2019. Political Geology. Active stratigraphies and the making of life, London, Palgrave.

Braun B., 2000. Producing vertical territory: geology and governmentality in the late Victorian Canada, Ecumene, 7, 7-46, http://www.jstor.org/stable/44252276.

Bridge G., 2013. Territory now in 3D!, Political Geography, 34, 55-57.

Chailleux S., 2019. Strategic ignorance and politics of time: how expert knowledge framed shaled gas policies, Critical Policy Studies, https://doi.org/10.1080/19460171.2018.1563556.

Chateauraynaud F., Debaze J., 2017. Aux bords de l'irréversible. Sociologie pragmatique des transformations, Pétra éditions.

Clark N., 2013. Geoengineering and geologic politics, Environment and Planning A, 45, 2825-2832, https://doi. org/10.1068/a45646.

Clark N., 2016. Politics of Strata, Theory, Culture \& Society, 34, 2-3, 211-231, https://doi.org/10.1177/0263276416667538.

Dalby S., 2013. The geopolitics of climate change, Political Geography, 37, 38-47, http://doi.org/10.1016/j.pol geo.2013.09.004.

Delborne J., Hasala D., Wigner A., Kinchy A., 2020. Dueling metaphors, fueling futures: "Bridge fuel" visions of coal and natural gas in the United States, Energy Research and Social Sciences, 61, https://doi.org/10.1016/j.erss.2019.101350.

Donsimoni M., 1981. Le bassin houiller lorrain. Synthèse géologique, Orléans, Éditions du BRGM.

Dewey J., 1927. The Public and its Problems: An Essay in Political Inquiry, Chicago, Gateway.

Dewey J., 1929. The quest for certainty: a study of the relation of knowledge and action, New York, Putnam.

Elden S., 2013. Secure the volume: Vertical geopolitics and the depth of power, Political Geography, 34, 35-51, https://doi. org/10.1016/j.polgeo.2012.12.009.

Gigandet A., 2002, Introduction, in Lucrèce, De la nature des choses, trad. B. Pautrat, Paris, Librairie générale française.

Hamblin J., 2008. Poison in the well. Radioactive waste in the oceans at the dawn of the nuclear age, Rutgers University Press.
IEA, 2012. Golden Rules for a Golden Age of Gas. World Energy Outlook, Special Report on Unconventional Gas.

IFP, 2015. Audit des réserves et ressources estimées de gaz naturel, Permis Bleue Lorraine, 30 septembre 2015.

Labussière O., Nadaï A., 2018. How to inquire about energy transition processes?, in Labussière O., Nadaï A. (Eds), Energy Transitions. A sociotechnical inquiry, London, Palgrave.

Lehman J., 2013. Volumes beyond volumetrics: A response to Simon Dalby's 'The Geopolitics of Climate Change', Political Geography, 37, 51-52, https://doi.org/10.1016/j. polgeo.2013.09.005.

Lucrèce, 2002. De la nature des choses, trad. par B. Pautrat, Paris, Librairie générale française.

Marres N., 2012. Material Participation: Technology, the Environment and Everyday Publics, London, Palgrave.

Massey D., 2005. For Space, London, Sage.

McCormack D., 2015. Envelopment, exposure and the allure of becoming elemental, Dialogues in Human Geography, 5, 1, 85-89, https://doi.org/10.1177/2043820614565875.

Neyrat F., 2017. La part inconstructible de la terre. Critique du géo-constructivisme, Paris, Seuil.

Petroleum Resources Management System, 2011. Guidelines for application of the Petroleum resources management system.

Rolston J.S., 2013. The politics of pits and the materiality of mine labor: Making natural resources in the American west, American Anthropologist, 115, 4, 582-594, https://doi.org/ 10.1111/aman.12050.

Serres M., 1977. La naissance de la physique, Paris, Éditions de Minuit.

Stein M., 2019. La controverse du « gaz de charbon » en France (2006-2018) : conflits de nomination et mise en question de la neutralité de l'expertise, Mots. Les langages du politique, 119, 1, 69-85, https://doi.org/10.4000/mots.24390.

Steinberg P., Peters K., 2015. Wet ontologies, fluid spaces: giving depth to volume through oceanic thinking, Environment and Planning D, 33, 247-264, https://doi.org/10.1068/d14148p.

Squire R., 2017. "Do you dive?”: Methodological considerations for engaging with 'volume', Geography Compass, 11, 7, e12319, https://doi.org/10.1111/gec3.12319.

Whatmore S., 2002. Hybrid Geographies, London, Sage.

Citation de l'article: Labussière O. Un «volume» incertain: géologie politique de l'exploration du gaz de charbon en Lorraine (France). Nat. Sci. Soc. 29, S69-S80. 